\title{
Weighting of different orientation sources in conflict experiments in Blattella germanica (L.), Dictyoptera: Blattellidae
}

\author{
COLETTE RIVAULT and LAURENT DABOUINEAU \\ Université de Rennes I, Rennes, France
}

\begin{abstract}
Control exerted by different categories of spatial information on short-distance return to shelter by young cockroach larvae and weighting of different information sources in conflict situations were investigated. In the absence of landmark cues, larvae preferred information given by path integration with kinesthetic cues over scototactic cues. When two landmark cues with a particular angular position between them were shifted $180^{\circ}$, larvae relied on these learned landmarks provided that their relative angular position was kept unchanged. When only one of the landmark cues was shifted $180^{\circ}$, larvae preferred to use the path integration system. When larvae could not use path integration cues, a modified configuration of the landmarks disturbed them. They relied on scototactic cues, although some seemed to rely on either one of the landmarks to find their shelter. Cockroaches could change the order of importance that they normally attributed to certain classes of spatial information (in increasing order: scototaxis, path integration, and learned visual landmarks) in relation to ongoing conditions.
\end{abstract}

Spatial orientation implies the integration of different information sources; that is, external cues and information of an internal navigation system that usually are consistent with each other. Insects use different sources of spatial information to navigate within their familiar home range, relying on nonlearned reactions to stimuli, for example, taxes (Kuhn, 1919) and genetically determined stimulus preferences (Papi \& Tongiorgi, 1963; Pardi, 1960; Ugolini \& Scapini, 1988). Cockroaches and crickets are spontaneously attracted to dark areas - that is, they exhibit positive scototaxis (Campan, Beugnon, \& Lambin, 1987; Darchen, 1954). Insects may also rely on memorized stimuli, based on either short-term memory or on long-term memory (Beugnon, 1986).

Path integration information is stored in short-term memory and is available only within a given sequence of locomotor activity. By means of a path integration system, an animal is able to direct its locomotion without using landmarks but by making use of information provided by its own previous movement sequences, or positions, which are transduced by exoskeleton sense organs (Mittelstaedt, 1983; Seyfarth, Hargenvioder, Ebbes, \& Barth, 1982; Wehner \& Wehner, 1986). Kinesthetic cues provide the animal with a continuous egocentric representation of its position relative to its starting point, including direction and distance (Seguinot, Maurer, \& Etienne, 1993; Seyfarth \& Barth, 1972). Celestial cues also contribute to the path integration system (Wehner, 1983) in some species, such as bees or ants. Stable environ-

Correspondence should be addressed to C. Rivault, Universite de Rennes I, Laboratoire d'Ethologie, CNRS URA 373, Campus de Beaulieu, 35042 Rennes Cedex, France. mental cues allow a subject to learn spatial relationships in its environment on a long-term basis by means of conspicuous landmarks.

An animal has to relate changes in the perception of different cues to its own movement to organize its visual environment. The correlation between the preceding movement and the expected changes in the visual field may reinforce the recognition of a given place (Etienne, Joris, Maurer, \& Teroni, 1990). During locomotion path integration, the internal navigation system (O'Keefe \& Nadel, 1978) must interact with external spatial cues that the animal encounters while moving through familiar space, as in updating via piloting (Gallistel, 1990). Usually pieces of information provided by different types of cues (internal cues and landmark cues) reinforce each other to help the animals to make the right decision. Weighting is the expression of the intensity with which an animal responds to one stimulus relative to another (Schöne, 1984). This response can vary among individuals and/or over time for the same individual (Etienne et al., 1990). According to the distance from the goal and the environmental conditions, animals can switch from one orientation system to the other, as observed in ants (Wehner, 1983) or amphipods (Ugolini, Scapini, \& Pardi, 1986).

The aim of this study was to investigate the control exerted by different categories of spatial information on short-distance return to shelter in the cockroach Blattella germanica (L.) (Dictyoptera: Blattellidae) and to analyze the weighting of different information sources in conflict situations.

Under our standard test conditions, the insect proceeds from its shelter (at the box periphery) to a food source (at the center of the box) and then returns to the shelter after a disturbance. As soon as larval cockroaches hatch, they 
are mobile. Larvae have to search for food and shelter, which are often located in different areas. Survival is likely to be enhanced if the larvae are able to navigate accurately and efficiently between these different areas. Young larvae are able to learn the conspicuous landmark cues of their environment in 2 or 3 days (Dabouineau \& Rivault, 1995).

Previous work has shown that two types of cues are used by cockroaches to return to their shelter: path integration with kinesthetic cues and memorized visual landmarks. Either type of cue is sufficient to allow cockroaches to return to the shelter, but orientation has been shown to be more precise when both types of cues can be used simultaneously (Dabouineau, 1992; Dabouineau \& Rivault, 1994). In dim red light (no visual information), a shake plus a rotation of the food dish where cockroaches are feeding distorts or renders kinesthetic cues inaccurate; cockroaches are unable to find their shelter and escape randomly. When the disturbance given to the food dish is a shake without a rotation, cockroaches orient correctly toward their shelter using a path integration process (Dabouineau \& Rivault, 1994). Odors of conspecifics are not used to locate food (Rivault \& Cloarec, 1992) or to return to shelter (Dabouineau, 1992). Since Blattella germanica is a species that lives strictly indoors, celestial cues are not appropriate cues to study.

In three series of experiments, we examined responses to three types of conflict situations: (1) scototactic versus kinesthetic cues, (2) kinesthetic versus visual cues, and (3) unreliable visual versus kinesthetic cues.

Scototaxis is understood as direct attraction toward black targets or objects in the environment (Campan \& Medioni, 1963; Jander, 1971); landmark orientation or use of visual cues refers to the use of a black target to find a goal (shelter, in our experiments) that is different from the target itself, and kinesthetic orientation refers to the use of internal cues related to previous movements of the body to find a goal (Wehner \& Wehner, 1986).

\section{METHOD}

\section{Subjects}

All larvae in one box came from the same oothecae deposited in the shelter the day before hatching (Tanaka, 1976). Animals hatched in the shelter of their test box and the 30 to 40 sister larvae were not marked to avoid any disturbance and handling. They were left in their box until the test, which occurred when they were 4 days old. The first instar lasts 6 to 7 days under these breeding conditions. During an instar, foraging and locomotory activity increase gradually over the first days, are maximum in the middle of the instar, and decrease before the molt. Day 4 was chosen because it is in the middle of the first instar (Dabouineau \& Rivault, 1988).

Each box was used only once so that individual larvae could not be tested several times. The number of larvae tested per box depended on the number of larvae present on the food dish at the time of the test, usually 5 or 6 . When a food dish was disturbed all the larvae present on the food escaped independently. The presence of other larvae did not influence escape behavior. Individual routes were followed on videotape and data for each cockroach larvae were analyzed independently. Tests were replicated until a sufficient number of larvae had been tested under each experimental condition.
Cockroaches exhibit a circadian activity rhythm synchronized by the light:dark cycle, with a maximum activity peak during the first night hours (Rivault, 1986). Tests were performed during the dark phase of the light:dark cycle. Intensity during the light phase was $90 \mathrm{~W} / \mathrm{m}^{2}$ and $3.8 \mathrm{~W} / \mathrm{cm}^{2}$ during the dark phase. The presence of some light during the dark phase of the light:dark cycle in the completely light-proof experimental rooms was necessary to allow the larvae to distinguish conspicuous landmark cues.

\section{Apparatus}

Animals were housed in square plastic boxes $(24 \times 24 \times 8 \mathrm{~cm})$ without a lid to facilitate handling and performing tests. An electric barrier around the top of the box gave animals only a mild shock and prevented them from escaping. In the middle of each vertical side was a rectangular black cardboard shelter $(6 \times 4 \times 1 \mathrm{~cm})$. Only one of them had a small entrance on its lower side, placed so that it could not be seen from the center of the box. The presence of four identical shelters, one at each of the cardinal directions, prevented the larvae from using the open shelter as a landmark. Water (a soaked piece of cotton wool) was placed near the side opposite the shelter. Food was placed in a small round plastic dish ( $3 \mathrm{~cm}$ in diameter and $0.6 \mathrm{~cm}$ high) in the center of the box (Figure 1). Ten boxes at a time were placed in the experimental room. Room temperature was $26^{\circ} \mathrm{C}$ and the photoperiod was $12: 12-\mathrm{h}$ light:dark. A high-sensitivity video camera (CCD Philips, 0.01 Lux), fixed above the boxes, was used to film the movements of the animals in the boxes during the tests.

\section{Procedure}

Light conditions and visual landmark cues. In all the experiments, the four black shelters (one open, three fake) were present as were the targets. In Experiments 1 and 2, a white nondirectional light $\left(3.8 \mathrm{~W} / \mathrm{m}^{2}\right)$ was on permanently, even during the dark phase of the light:dark cycle, to allow the larvae to distinguish their surroundings. The only salient targets were the four black shelters, which gave no indication of the location of the open shelter.

In Experiments 3 through 7, two types of visual landmarks were used. The first type of landmark was given by a white light $\left(3 \mathrm{~W} / \mathrm{m}^{2}\right)$ directed toward one side of the experimental room, the position of which could be varied. The light itself was not visible. The illuminated side of the room was considered a distal landmark (Morris, 1981). The second type of landmark was a small black square of cardboard glued on the top edge of the test box on the left side of the open shelter. It was called a proximal landmark. These two landmark cues provided directional information that could be used by the larvae to locate the shelter.

Disturbance and kinesthetic cues. A test measured the escape direction of the larvae from the food dish following a disturbance. The predicted escape direction was the shelter direction. Two types of disturbance could be given by the investigator to the food dish:

1. A translation movement several centimeters in amplitude and back to the initial place, without a rotation, was called a shake. This stress has been shown to induce escape toward the shelter without interfering with orientation performance (Dabouineau \& Rivault, 1994). This manipulation does not affect the accuracy of path integration by means of kinesthetic cues or visual orientation by means of learned landmark cues.

2 . The food dish was rotated as well as given a translation movement. This disturbance was called shake + rotation. Kinesthetic cues became inaccurate after a passive rotation of the body of the animals. Feeding animals were rotated with the food dish. The magnitude of the rotation was the same for all individuals (and all dishes). The rotation, always given by the same experimenter, was approximately $120^{\circ}$ clockwise. Previous results have shown that this type of disturbance prevents larvae from orienting correctly toward the shelter when path integration with kinesthetic cues is disturbed and visual cues are absent (Dabouineau, 1992; Dabouineau \& Rivault, 1994). 

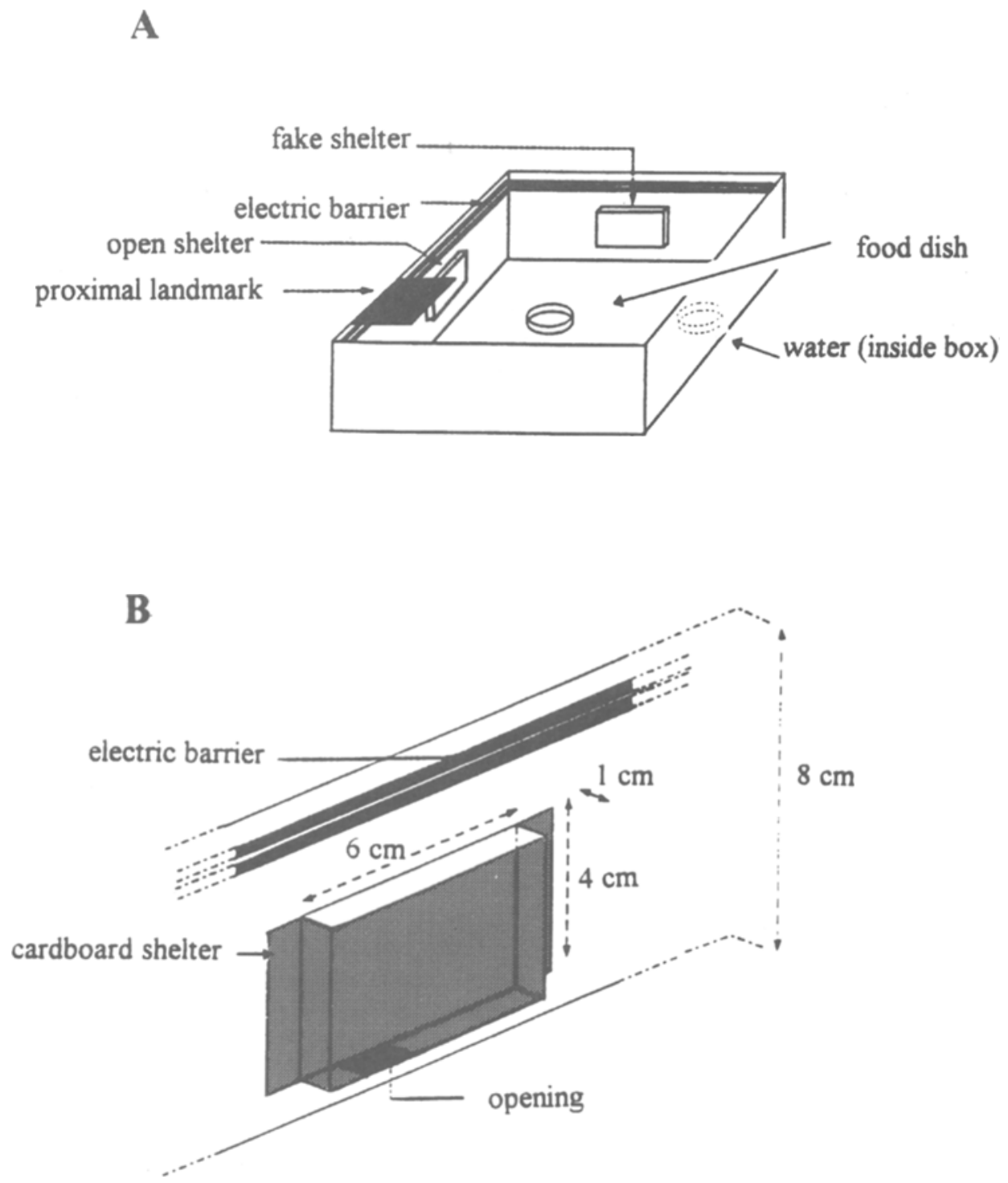

Figure 1. Panel A: experimental test box, with one open shelter near the proximal landmark and three fake shelters in the other cardinal directions. Panel B: details of the open shelter.

Three series of experiments. In Experiments 1 and 2, four shelters were placed in the test box. Compared to the fake shelters, the open shelter gave no cue the cockroach could detect from the center of the test box (Figure 1). Uniform nondirectional lighting was present in the room (Figure 2). Until the 3rd day, larvae had no landmark cues to orient their return to the shelter. Test conditions on the 4th day were as follows. In Experiment 1, larvae still had no landmark cues, and they were submitted to a shake + rotation in the food dish, which meant that kinesthetic cues became inaccurate after this stress. In Experiment 2, the experimental conditions were the same except that the stress given to the larvae consisted of a simple shake that did not distort kinesthetic cues.

In Experiments 3 and 4, larvac were given two types of landmark cues to guide their return to the shelter during their first 3 days of life. A black landmark (proximal landmark) was added near the open shelter and a light was directed toward one side of the experimental room (distal landmark). During the test, the position of both the directional light and the additional landmark were shifted $180^{\circ}$. In Experiment 3, a shake + rotation disturbed kines- thetic cues. In Experiment 4, kinesthetic cues were not distorted since larvae were submitted only to a shake.

In Experiments 5 and 6, the initial experimental conditions were the same as those in Experiments 3 and 4. Larvae had two types of visual landmark cues to rely on to find their shelter direction. Changing the position of one type of cue in relation to the other was intended to help us to determine whether one type of landmark was more important than the other. Kinesthetic cues were disturbed with a shake + rotation of the food dish. In Experiment 5 , the proximal landmark was kept in the same position throughout the test while the distal landmark was shifted $180^{\circ}$, in the opposite direction. In Experiment 6, the distal landmark was kept unchanged and the proximal landmark was shifted $180^{\circ}$ and placed near the fake shelter opposite the open shelter.

In Experiment 7, the initial experimental conditions were the same as those in Experiments 5 and 6. During the test, visual landmark cues were the same as those in Experiment 6 (distal landmark unchanged, proximal landmark shifted $180^{\circ}$ ). Kinesthetic cues were kept accurate by submitting larvae to a shake only. 


\begin{tabular}{|c|c|c|}
\hline Experiment & & \\
\hline Visual cues until 3rd day & & \\
\hline vest conditions \\
visual cues & & \\
\hline Results & & \\
\hline & & \\
\hline
\end{tabular}

Figure 2. Experiments 1 and 2; scototactic versus kinesthetic cues. The experimental test boxes, under a uniform lighting, presented one open shelter (open rectangle) and three fake shelters (closed rectangles), giving off no cue the larvae could detect. During the test in Experiment 1 , a $120^{\circ}$ clockwise rotation of the food dish induced inaccuracy of kinesthetic cues, and larvae were unable to orient toward the open shelter. In Experiment 2, kinesthetic cues were kept accurate and larvae oriented significantly toward the open shelter. Legend: Each dot around the circle indicates individual orientation of one larva. The open square inside the circle indicates open shelter direction set at $90^{\circ}$. Closed squares indicate fake shelters. The arrow inside the circle represents mean vector. $N$, number of larvae tested; $r$, mean vector length; $\Phi$, mean direction angle; $p$, significance level of Rayleigh test; $\delta$, angular deviation.

Data analysis. Escape directions following the disturbance of each individual present on the food dish were recorded and analyzed statistically. Directions were measured when animals reached the edge of a circle $12 \mathrm{~cm}$ in diameter. Directions were measured in angles ranging from $0^{\circ}$ to $360^{\circ}$ and analyzed as circular variables. The theoretical escape direction given by the shelter was set at $90^{\circ}$. Graphical presentations of data followed the rules and definitions given by Batschelet (1981). Applying vector algebra, we calculated the mean vector with its two polar coordinates, $r$ (length ranging from 0 to 1 ) and $\Phi$ (mean orientation angle relative to expected direction), and its confidence interval $\delta$ for each set of data. Significance was estimated using Rayleigh tests, which are goodness-of-fit tests for randomness.

The hypothesis tested was that cockroaches faced with four identical target shelters would be able to find their open shelter using different orientation cues given during the experiments. This hy- pothesis was tested against the null hypothesis that they would not be able to find it. If the observed distribution of data was unimodal, we reasoned, the significance of the Rayleigh test would prove not only one-sidedness but also a concentration of directions around the mean direction, which could be called the preferred direction.

If and only if samples did not show one-sidedness, quadrimodality of data was tested with four modes $90^{\circ}$ apart from one another around the circle in relation to the four identical target shelters. The Rayleigh test can still be used with these data provided that the quadrimodal distribution of the data is converted into a unimodal distribution. We quadrupled observed angles and reduced multiples modulo $360^{\circ}$ before applying the test (Batschelet, 1981). The tested hypothesis was then that cockroaches would orient significantly toward the four shelters against the null hypothesis that they would orient randomly. 
If and only if samples did not show one-sidedness, bimodality of data (in Experiments 5 and 6 ) was tested with two modes $180^{\circ}$ apart from each other around the circle in relation to the two visual landmarks (one was shifted $180^{\circ}$ from its initial position). The Rayleigh test can still be used with the data provided that the bimodal distribution of the data are converted into a unimodal distribution. Observed angles were doubled and multiples reduced modulo $360^{\circ}$ before we applied the test. In this case, the tested hypothesis was that cockroaches would use preferentially either of the two visual landmark cues (changed or unchanged) to orient toward a shelter. The null hypothesis was that they would use neither of the landmark cues to orient.

\section{RESULTS}

\section{Scototactic Versus Kinesthetic Cues}

In Experiment 1, there were four identical equally attractive black targets in the test box. As larvae were exposed to a shake + rotation, kinesthetic cues became inaccurate after this stress. The Rayleigh test performed on orientation data did not reveal a preferred direction (Rayleigh test, $p>.05$ ). However, the diagram (Figure 2) suggested that four directions corresponding to the four shelters were preferred and that the distribution of the sample appeared quadrimodal. After the angles were multiplied by 4 , the distribution became significant by the Rayleigh test at the .01 level. These findings suggest that cockroaches were unable to find their shelter. They headed toward the four directions given by the black shelters. Because cockroaches are attracted to dark objects (Campan \& Medioni, 1963; Jander, 1971), larvae took the directions given by scototactic cues. If kinesthetic cues had been used, they would have been able to find the right shelter, and our results did not indicate that.

In Experiment 2, under the same experimental conditions except that kinesthetic cues were kept accurate after the stress, the results were completely different. Larvae headed significantly toward the open shelter (Rayleigh test, $p<.05)$. Mean orientation angle $\left(\Phi=75^{\circ}\right)$ matched the $90^{\circ}$ theoretical escape direction given by the shelter position. With the help of kinesthetic cues, therefore, larvae were able to orient significantly toward the open shelter despite the presence of the three fake shelters at the other three cardinal compass directions (Figure 2), which somehow lost their attractive power.

These experiments did not describe a real conflict situation (contradiction between several sets of information) because the black color of the shelter reinforces the natural attraction of cockroach to dark areas. Nevertheless in Experiment 1, larvae were attracted toward all black targets that were present. They used scototactic cues to find a refuge. In Experiment 2, they chose between four similarly attractive targets and used kinesthetic cues to do so. Kinesthetic cues seemed to be preferred over scototactic cues in this case.

\section{Kinesthetic Versus Visual Cues}

In Experiment 3, larvae were given both a proximal and a distal landmark cue with a particular angular position between them to orient toward their shelter. After a $180^{\circ}$ shift of both landmark cues from their initial position, which kept accurate their spatial relationship and a disturbance of path integration cues with a shake + rotation, larvae did not go toward the open shelter but oriented in the opposite direction, following the conspicuous landmark cues they had learned (Rayleigh test, $p<$ $.001)$. The confidence interval for the mean orientation angle $\left(\Phi=260^{\circ}\right)$ included the $270^{\circ}$ theoretical escape direction given by the new position of the landmark cues (Figure 3).

In Experiment 4, experimental conditions were similar to those in Experiment 3, except that kinesthetic cues were kept accurate. Larvae oriented in the direction opposite the open shelter following the landmark cues (Rayleigh test, $p<.05)$. The mean orientation angle $(\Phi=$ $255^{\circ}$ ) matched the $270^{\circ}$ theoretical escape direction (Figure 3 ). Stimuli perceived by larvae were contradictory. Kinesthetic cues indicated the direction of the open shelter and were correct. Visual landmark cues indicated the direction opposite the shelter, but were false. With contradictory information, a significant number of larvae favored learned visual landmark information over kinesthetic information. The comparison between Experiments 3 and 4 indicated that cues given by path integration in Experiment 4 did not prevent larvae from following landmark cues.

\section{Unreliable Visual Versus Kinesthetic Cues}

Initial experimental conditions in Experiments 5 and 6 were the same as those in Experiments 3 and 4. Larvae had the same two types of landmark cues to rely on to find their shelter direction. During the test, kinesthetic cues were disturbed with a shake + rotation and one type of visual landmark was shifted $180^{\circ}$, thus modifying the spatial relationship between landmarks.

In Experiment 5, the proximal landmark was kept in the same position throughout the test while the distal landmark was shifted $180^{\circ}$ to the opposite direction. A Rayleigh test performed on the orientation data did not reveal significant orientation $(p>.05)$. The larvae were not able to find their shelter when they had to rely exclusively on two landmarks with changed relative angular positions (also they cannot rely on path integration cues).

The diagram suggested that four directions were preferred (Figure 4). After the angle data were multiplied by 4 , a Rayleigh test indicated significance at the .01 level $\left(\Phi=80^{\circ} \pm 18^{\circ}, r=.20\right)$. Larvae oriented significantly toward the four shelters, suggesting that they were using scototactic cues.

After the angle data were multiplied by 2, a Rayleigh test indicated significance at the .01 level $(r=.22)$. Larvae oriented along an axis $\left(\Phi=96^{\circ} \pm 35^{\circ}\right)$ given by the unchanged proximal landmark and the changed distal landmark. Larvae would have relied on either one of the landmarks (changed or unchanged) to find their shelter. These test results do not allow us to say if the larvae chose one landmark rather than the other, only that they preferentially chose two shelters $180^{\circ}$ apart along the axis given by the landmarks, out of the four shelters present. 


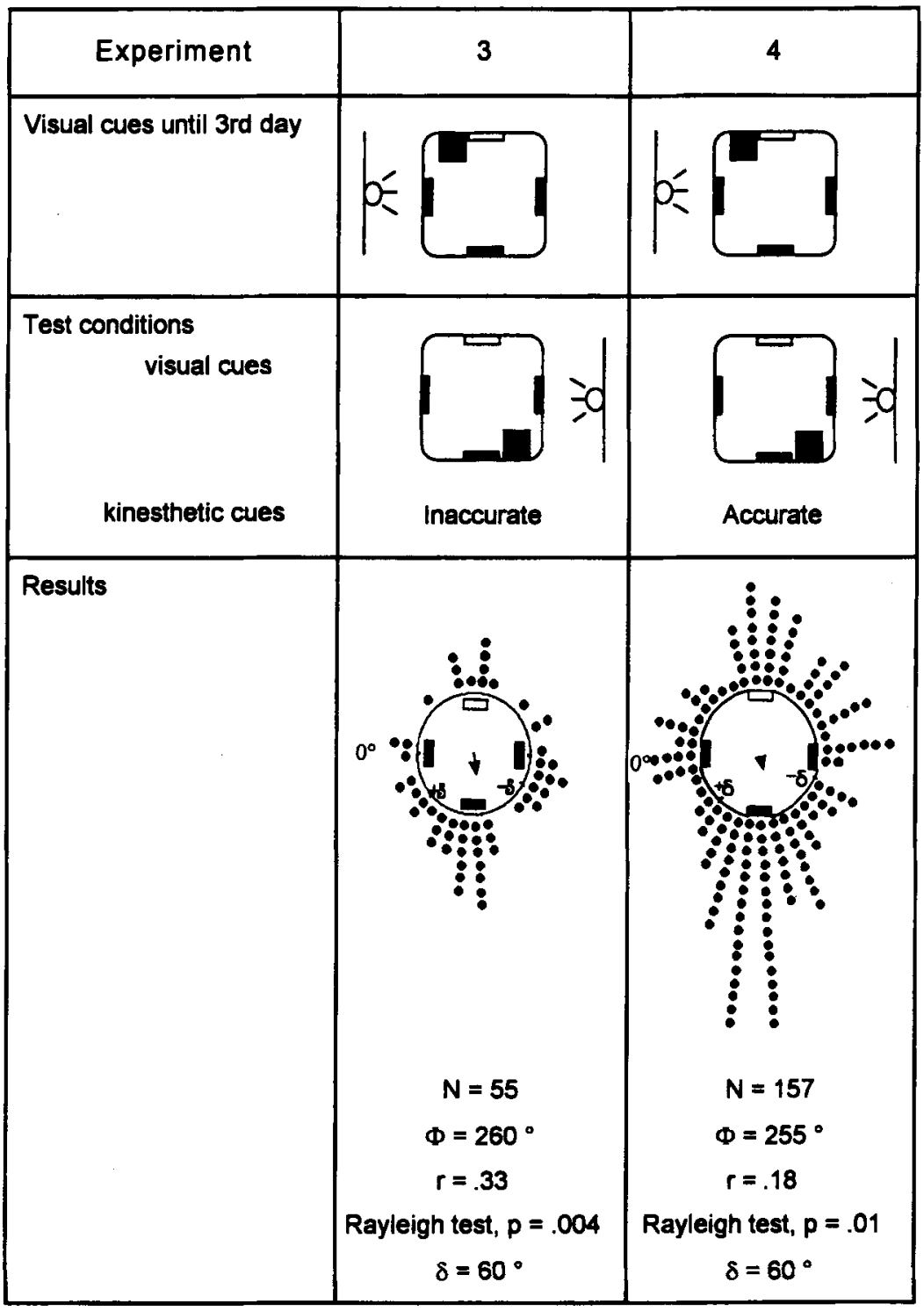

Figure 3. Experiments 3 and 4; kinesthetic versus visual cues. Experimental boxes presented one open shelter near the black proximal landmark and three fake shelters. An additional white light was directed toward one side of the experimental room and was considered a distal landmark. During the test, in Experiment 3, both proximal and distal landmarks were shifted $180^{\circ}$ (their relative position being kept). In addition, a $120^{\circ}$-clockwise rotation of the food dish induced inaccuracy of kinesthetic cues. Larvae used the learned landmark cues and headed in the direction opposite the open shelter. In Experiment 4, kinesthetic cues were kept accurate and larvae chose the landmark cues to orient correctly toward the shelter. $N$, number of larvae tested; $\Phi$, mean direction angle; $r$, mean vector length; $p$, significance level of Rayleigh test; $\delta$, angular deviation.

In Experiment 6, the distal landmark was kept unchanged and the proximal landmark was shifted to the direction opposite the open shelter during the test. There was again no preferred orientation toward the open shelter (Rayleigh test, $p>.05$ ) (Figure 4). As in Experiment 5, larvae were not able to find their shelter with landmarks when their relative positions had been modified.
The diagram suggested that four directions were preferred (Figure 4). After the angle data were multiplied by 4 , a Rayleigh test indicated significance at the .01 level $\left(\Phi=87^{\circ} \pm 16^{\circ}, r=.38\right)$. Larvae oriented significantly toward the four shelter directions, suggesting that they were using scototactic cues.

After the angle data were multiplied by 2, a Rayleigh test indicated no preferred axis $(r=.16, p>.07)$. Larvae 


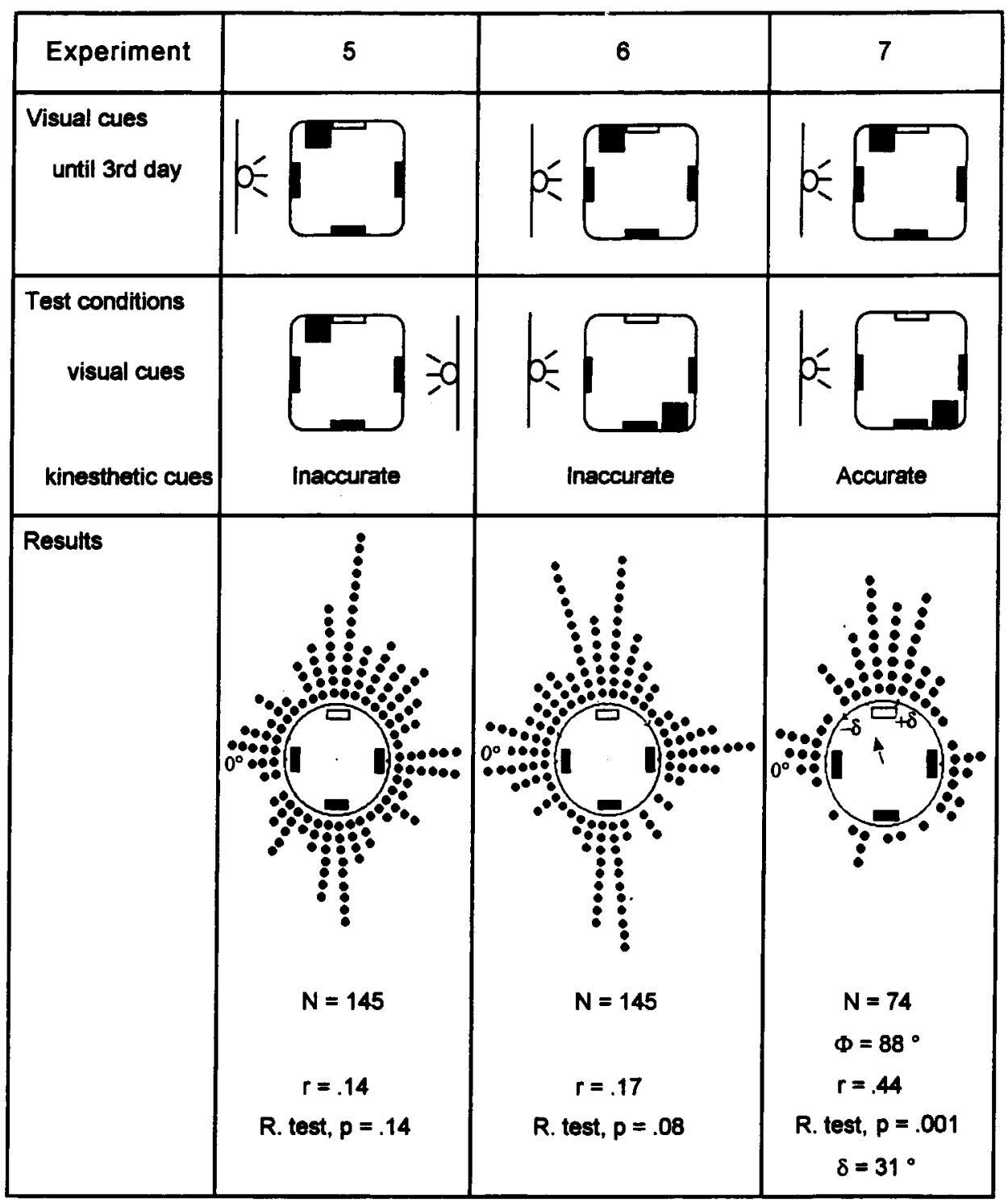

Figure 4. Experiments 5,6, and 7; unreliable visual versus kinesthetic cues. The initial experimental conditions were the same as in Experiments 3 and 4. During the test, in Experiments 5 and 6, kinesthetic cues were made inaccurate and one of the landmarks (proximal or distal) was shifted $180^{\circ}$. Larvae did not orient significantly toward a particular direction. In Experiment 7, kinesthetic cues were kept accurate and larvae were oriented toward the open shelter. $N$, number of larvae tested; $r$, mean vector length; $p$, significance level of Rayleigh test; $\delta$, angular deviation.

did not appear to have used either of the landmarks preferentially.

In Experiments 5 and 6, the distribution of directional responses indicated that the larvae's orientation was greatly disturbed. In both experiments larvae unable to use kinesthetic information seemed to have been disturbed by the changes in the relative spatial distribution of learned visual cues and appeared to rely mainly on scototactic cues to orient, although the data suggested that some larvae might have been able to orient along the axis given by the landmarks (changed and unchanged).
Experiment 7 replicated Experiment 6 except that kinesthetic cues were kept accurate by submitting larvae to a shake only. Larvae were able to orient toward the shelter (Rayleigh test, $p<.001$ ). The mean orientation angle $\left(\Phi=88^{\circ}\right)$ matched the $90^{\circ}$ shelter direction. In Experiment 7, larvae seemed to rely on kinesthetic cues over modified visual cues. We know from Experiment 6 that larvae did not orient significantly along the axis given by the two landmarks, and from Experiment 4 that larvae oriented using landmarks when their relative position was kept unchanged; furthermore, kinesthetic cues are 
sufficient to allow larvae to orient correctly (Dabouineau \& Rivault, 1994). Therefore, the results of Experiment 7 could be interpreted as reliance on kinesthetic cues.

\section{DISCUSSION}

These three series of conflict experiments provided interesting information on how different sources of information were weighted. In the first series of experiments, first instar cockroach larvae exhibited preference for information given by kinesthetic over scototactic cues. In Experiment 1, larvae relying on scototactic cues were attracted to four directions by four conspicuous targets. With a second source of information given by kinesthetic cues (Experiment 2), they were able to choose among these four directions and to orient toward the open shelter. Scototaxis is a nonlearned process and is described as a spontaneous tendency, present soon after hatching and lasting into adulthood (Campan, 1978; Schöne, 1984; Campan et al., 1987). Cockroaches actively orient their bodies toward a scototactic stimulus and go toward it without any memorization as long as they have not been deprived of the stimulus during development (Charii, 1991). Path integration is also present from birth and gives rise to short-term memorization of a set of information through kinesthetic cues. This memory is reset at zero once the animal is back at its starting point or what it thinks is its starting point (Wehner \& Wehner, 1986). Our results showed clearly that preference was given to information derived from internal signals correlated with the subject's changes in location and orientation in space, also known as kinesthetic information, rather than to information given by external cues. In these experiments, path integration with kinesthetic cues seemed to provide more precise and more reliable information and was used in preference to spontaneous scototaxis. This result could also be interpreted as a choice of a given direction among the four possible directions, the two types of information being used in conjunction and giving rise to a more precise orientation.

In the second series of experiments, larvae had to deal with conflicting information coming from learned visual landmark cues and kinesthetic cues. These two sources of directional information indicated opposite directions. Under these conditions, cockroach larvae relied on landmark cues rather than on kinesthetic cues. When learned visual landmark cues acted in conjunction with path integration, an increase in clustering around the expected orientation direction was observed (Dabouineau \& Rivault, 1994). Sandhoppers can also use two sets of information in conjunction to orient toward the sea-visual landscape cues and path integration with solar orientation (Ugolini et al., 1986). Unlike kinesthetic cues, landmark cues must be stored in long-term memory. As a larva matures, its individual experience increases (Cloarec \& Rivault, 1991). Maturation is also associated with increased contrast sensitivity and form perception (Campan et al., 1987).
With path integration, the animal uses a self-centered system of coordinates to keep continuous track of its own position relative to home. Navigational errors accumulate as long as the animal continues exploring (Mittelstaedt \& Mittelstaedt, 1973; Wehner \& Wehner, 1986). With learned cues, there may be a reduction in navigational errors. Therefore, cockroach larvae may have chosen the more reliable set of information, which required them to refer to long-term memory rather than to shortterm memory. Ugolini et al. (1986) found that under conflicting conditions, sandhoppers no longer oriented correctly toward the sea, deviating from the mean direction while dispersion increased up to total dispersion when learned local features were in total contrast to solar orientation. Sandhoppers, like cockroaches, appear confused and unable to switch to one type of cue to the detriment of the other.

The third series of experiments revealed two major results. First, when the learned landmark configuration was altered and kinesthetic cues were absent, larvae relied on positive scototaxis to orient toward conspicuous targets, although some larvae seemed to favor the axis made by the new position of the two landmarks. Second, when given path integration information, the animals preferred it over the modified landmark information. With a changed landmark configuration, long-term learned landmark cues were unreliable. Then, larvae switched to the more reliable information source they can use, namely the path integration system or scototaxis.

Walking cockroaches are capable of changing the order of importance that they normally attribute to different types of spatial information in relation to the apparent recognizability of information when ongoing conditions are modified. Similar phenomena have been observed in flying bees, which paid more attention to landmarks recognized as being close to their goal. Distant landmarks are not ignored but are weighted less heavily than close ones (Cheng, Collett, Pickhard, \& Wehner, 1987). However, in these bee experiments, only conflicts between learned visual orientation cues were analyzed, whereas in the cockroach and sandhopper experiments, the use of different orientation systems in the presence of conflicting cues was investigated. In hamsters (Etienne, Lambert, Reverdin, \& Teroni, 1993) the relative weight of the familiar visual cues compared to path integration cues increased in proportion to the state of conflict the subjects were exposed to; however, hamsters could learn to rely on path integration rather than visual cues. The preference of rats (Margules \& Gallistel, 1988) for different types of directional information may be explained by differences in the reliability and stability of the chosen systems. Observed orientation presumably reflects the relative weight that is given to each source of information. The weights derive from uncorrelated spatial information and the hierarchy of sources that might correlate with their predictiveness. Cockroaches always switched to the more reliable set of information. Under normal conditions, learned spatial configurations of landmarks are 
preferred over other types of information, and they usually reinforce or sharpen information given by the other sources. Usually visual configurations and kinesthetic information remain correlated during a trip. Our experimental conditions revealed that cockroaches, like some vertebrates, will change the relative weighting of different types of directional information used for homing in relation to the reliability of the different types of cues.

\section{REFERENCES}

Batschelet, E. (1981). Circular statistics in biology. London: Academic Press.

Beugnon, G. (1986). Orientation in space. Toulouse: Privat.

Campan, R. (1978). L'utilisation des repères visuels terrestres dans l'orientation des insectes. Annales de Biologie, 17, 61-90.

Campan, R., Beugnon, G., \& Lambin, M. (1987). Ontogenetic development of behavior: The cricket visual world. Advances in the Study of Behavior, 17, 165-212.

Campan, R., \& Medioni, J. (1963). Sur le comportement "scototactique" du grillon Nemobius sylvestris Bosc. Compte Rendus des Séances de la Société de Biologie, 157, 1690-1695.

CHARII, F. (1991). La construction de l'univers visuel et le rôle de l'expérience individuelle chez le grillon Gryllus bimaculatus. Thèse de doctorat, Université Paul Sabatier, Toulouse.

Cheng, K., Collett, T. S., Pickhard, A., \& Wehner, R. (1987). The use of visual landmarks by honeybees: Bees weight landmarks according to their distance from the goal. Journal of Comparative Physiology, 161, 469-475.

Cloarec, A., \& Rivault, C. (1991). Age related changes in foraging in the German cockroach (Dictyoptera: Blattellidae). Journal of Insect Behavior, 4, 661-673.

DABOUINEAU, L. (1992). Utilisation de l'habitat, retour au gite et représentation de l'espace chez Blattella germanica (L.) (Dictyoptera, Blattellidae). Thèse de doctorat, Université de Rennes I, Rennes.

Dabouineau, L., \& Rivault, C. (1988). Nourriture et dispersion chez les jeunes larves de Blattella germanica (L.). Actes des Colloques Insectes Sociaux, 4, 307-315.

Dabouineau, L., \& Rivault, C. (1994). Spatial orientation in Blattella germanica (L.) larvae. Ethology, 98, 101-110.

Dabouineau, L., \& Rivault, C. (1995). Ontogenetic development of spatial orientation in first- and second-instar cockroach larvae (Blattella germanica (L.), Dictyoptera). Ethology, 100, 148-159.

DARCHEN, R. (1954). Stimuli nouveaux et tendance exploratrice chez Blattella germanica. Zeitschrift für Tierpsychologie, 12, 1-11.

Etienne, A. S., Joris, S., Maurer, R., \& Teroni, E. (1990). Enhancing the impact of visual extramaze cues in a spatial orientation task. Behavioral Brain Research, 38, 199-210.

Etienne, A. S., Lambert, S. J., Reverdin, B., \& Teroni, E. (1993). Learning to recalibrate the role of dead reckoning and visual cues in spatial navigation. Animal Learning \& Behavior, 21, 266-280.

GaLlistel, C. R. (1990). The organization of learning. Cambridge, MA: MIT Press, Bradford Books.

JANDER, R. (1971). Visual pattern recognition and directional orientation in insects. Annals of the New York Academy of Sciences, 188, 5-11.
KuHn, A. (1919). Die Orientierung der Tiere im Raum. Jena, Germany. Margules, J., \& Gallistel, C. R. (1988). Heading in the rat: Determination by environmental shape. Animal Learning \& Behavior, 16, 404-410.

MitTelstaedT, H. (1983). The role of multimodal convergence in homing by path integration. Fortschritte der Zoologie, 28, 197-212.

MittelstaedT, H., \& MitTelstaedt, M. L. (1973). Homing by path integration. In F. Papi \& H. G. Wallrahf (Eds.), Avian navigation (pp. 290-297). Berlin: Springer-Verlag.

MorRIS, R. G. M. (1981). Spatial localization does not require the presence of local cues. Learning \& Motivation, 12, 239-260.

O'KeEFE, J., \& NADEL, L. (1978). The hippocampus as a cognitive map. Oxford: Oxford University Press, Clarendon Press.

PAPI, F., \& TongIORGI, P. (1963). Innate and learned components in the astronomical orientation of Wolf-Spiders. Ergebnisse der Biologie, 26, 259-280.

PARDI, L. (1960). Innate components in the solar orientation of littoral amphipods. Cold Spring Harbor Symposia on Quantitative Biology, 25, 395-401.

RIVAULT, C. (1986). Behavioural circadian activity peak in social groups of Periplaneta americana (Dictyoptera). In Y. Quéinnec \& N. Delvolvé (Eds.), Behavioural rhythms (pp. 57-65). Toulouse: Privat.

Rivault, C., \& Cloarec, A. (1992). Foraging in Blattella germanica (L.) and aggregation pheromone. Etologia, 2, 33-39.

SCHÖNE, H. (1984). Spatial orientation. The spatial control of behavior in animals and man. Princeton, NJ: Princeton University Press.

Seguinot, V., Maurer, R., \& Etienne, A. S. (1993). Dead reckoning in a small mammal: The evaluation of distance. Journal of Comparative Physiology, 173, 103-113.

SeYfarTh, E. A., \& BarTh, F. G. (1972). Compound slit sense organs on the spider leg: Mechanoreceptors involved in kinesthetic orientation. Journal of Comparative Physiology, 78, 176-191.

Seyfarth, E. A., Hargenvioder, R., Ebbes, H., \& Barth, F. G. (1982). Idiothetic orientation of a wandering spider: Compensation of detours and estimates of goal distance. Behavioral Ecology \& Sociobiology, 11, 139-148.

TANAKA, A. (1976). Stages in the embryonic development of the German cockroach Blattella germanica (Blattaria, Blattellidae). Kontyu, Tokyo, 44, 512-525.

UgOLINI, A., \& SCAPINI, F. (1988). Orientation of the sandhopper $T a$ litrus saltator (Amphipoda, Talitridae) living on dynamic sandy shores. Journal of Comparative Physiology, 162, 453-462.

Ugolini, A., SCAPINI, F., \& PARDI, F. (1986). Interaction between solar orientation and landscape visibility in Talitrus saltator (Crustacea, Amphipoda). Marine Biology, 90, 449-460.

WeHNER, R. (1983). Celestial and terrestrial navigation: Human strategies - Insect strategies. In P. Huber \& H. Markl (Eds.), Neuroethology and behavioral physiology (pp. 366-381). Berlin: SpringerVerlag.

Wehner, R., \& Wehner, S. (1986). Path integration in desert ants. Approaching a long standing puzzle in desert navigation. Monitore Zoologico Italiano, 20, 309-331.

(Manuscript received July 3, 1995; revision accepted for publication July 25,1995 .) 